\title{
AN EXAMPLE CONCERNING THE YOSIDA-HEWITT DECOMPOSITION OF FINITELY ADDITIVE MEASURES
}

\author{
WOLFGANG HENSGEN
}

(Communicated by Dale Alspach)

\begin{abstract}
Let $\lambda$ be Lebesgue measure on the Lebesgue $\sigma$-algebra $\mathscr{L}$ of $I:=] 0,1[$. The author gives an example of a purely finitely additive measure $\varphi: \mathscr{L} \rightarrow[0,1]$ vanishing on $\lambda$-null sets such that $\int f d \varphi=\int f d \lambda$ for every bounded continuous function $f$ on $I\left(f \in C_{b}(I)\right)$. Consequently, $\lambda-\varphi \in L^{\infty}(\lambda)^{\prime}$ annihilates $C_{b}(I)$ and is not purely finitely additive, contrary to an assertion of Yosida and Hewitt.
\end{abstract}

To be in accordance with [HY], the scalar field is $\mathbb{R}$, although $\mathbb{C}$ could be used with insignificant changes in what follows. Also, only bounded real-valued set functions are considered. A finitely additive measure $\varphi \geq 0$ on an algebra of sets is called purely finitely additive (p.f.a.) if every $\sigma$-additive measure $\mu$, $0 \leq \mu \leq \varphi$, is zero. If the requirement $\varphi \geq 0$ is dropped, $\varphi$ is called p.f.a. if the positive and negative variations $\varphi_{+}$and $\varphi_{-}$are both p.f.a. Every finitely additive measure can be uniquely written as $\mu+\varphi$, where $\mu$ is $\sigma$-additive and $\varphi$ is p.f.a. This is the content of the Yosida-Hewitt decomposition theorem [HY, 1.24]. In later sections of this fundamental work on finitely additive measures, the authors study the bounded linear functionals on $L^{\infty}(\lambda)$. Those are easily identified as the finitely additive measures on $\mathscr{L}$ vanishing on $\lambda$-null sets [HY, 2.3]. Such a measure $\varphi$ is p.f.a. if and only if it is concentrated on sets of arbitrarily small $\lambda$-measure, where $\varphi$ is called concentrated on $E \in \mathscr{L}$ if $|\varphi|(I \backslash E)=0$ [HY, 3.1].

Let us turn to the construction of the example announced in the abstract. For any $t_{0} \in I$ there exists a positive (hence bounded) linear functional $\varphi_{0}$ on $L^{\infty}(\lambda)$ with $\varphi_{0} \mid C_{b}(I)=\delta_{t_{0}}:=$ point evaluation at $t_{0}$. Just apply the HahnBanach theorem to find a linear functional below the sublinear functional $f \mapsto$ ess $\lim \sup _{t \rightarrow t_{0}} f(t):=\lim _{\varepsilon \rightarrow 0}$ ess $\sup _{t_{0}-\varepsilon \leq t \leq t_{0}+\varepsilon} f(t)$ on $L^{\infty}(\lambda)$. [One could even find a character of $L^{\infty}(\lambda)$ extending $\delta_{t_{0}}$ (see, e.g., [IT, p. 107], but I will not need this.] Any such $\varphi_{0}$ is concentrated on every neighbourhood $U$ of $t_{0}$ (in particular p.f.a.). To see this, take any $f \in C_{b}(I), f \leq 1_{U}, f\left(t_{0}\right)=1$. Then $1=\delta_{t_{0}}(f)=\varphi_{0}(f) \leq \varphi_{0}\left(1_{U}\right) \leq \varphi_{0}(1)=1$, so $\varphi_{0}\left(1_{U}\right)=1$.

Received by the editors December 10, 1992.

1991 Mathematics Subject Classification. Primary 28A10, 28C15; Secondary 46E30, 46G99.

Key words and phrases. Finitely additive measures, Yosida-Hewitt decomposition.

This example is contained in the author's habilitation thesis $[\mathrm{H}]$. 
Now let $t_{n j}:=\left(j+\frac{1}{2}\right) 2^{-n}$ for $n \in \mathbb{N}, 0 \leq j \leq 2^{n}-1$, and $\varphi_{n j}$ be a positive functional on $L^{\infty}(\lambda)$ with $\varphi_{n j} \mid C_{b}(I)=\delta_{t_{n j}}$. Next, choose and fix a Banach limit Lim, that is a positive (hence bounded) linear extension of the "lim" functional over $\ell^{\infty}$. (Take a linear functional below limsup.) For $x=\left(x_{n}\right)_{n \in \mathbb{N}} \in \ell^{\infty}$ write $\operatorname{Lim}_{n \rightarrow \infty} x_{n}$ instead of $\operatorname{Lim}(x)$.

Define $\varphi(f):=\operatorname{Lim}_{n \rightarrow \infty} 2^{-n} \sum_{j=0}^{2^{n}-1} \varphi_{n j}(f)$ for $f \in L^{\infty}(\lambda)$. Obviously, $\varphi \in$ $L^{\infty}(\lambda)^{\prime}$ is positive and concentrated on every neighbourhood of the set of points $t_{n j}$; hence, $\varphi$ is p.f.a. Moreover, any $f \in C_{b}(I)$ arbitrarily extended over $[0,1]$ is Riemann integrable over $[0,1]$, so $\varphi(f)=\int_{0}^{1} f(t) d t=\int f d \lambda . \varphi(1)$ being 1 , the set function $\varphi(A)=\varphi\left(1_{A}\right), A \in \mathscr{L}$, has all the announced properties.

Finally, the difference $\lambda-\varphi \in L^{\infty}(\lambda)^{\prime}$ annihilates $C_{b}(I)$ but is not p.f.a. (uniqueness of the decomposition!), contrary to the last assertion of [HY, Theorem 3.4].

The careful reader might argue that Yosida and Hewitt consider $\mathbb{R}$ instead of $I$. However, note that their measure $\lambda$ is an (arbitrary) probability measure equivalent to Lebesgue measure on $\mathbb{R}$. Therefore, any both-way null setpreserving homeomorphism $I \rightarrow \mathbb{R}$ can be used for translation.

\section{NOTED ADDED IN PROOF}

After this note had been accepted for publication, I found that M. Valadier (Une singulière forme linéaire singulière sur $L^{\infty}$, Sém. d'Analyse Convexe Montpellier 1987, Expose no. 4) has (also) constructed a p.f.a. functional on $L^{\infty}(\lambda)$ which extends the functional $\lambda$ on $C[0,1](!)$. In this connection, see also the article by Y. A. Abramovich and A. W. Wickstead, Singular extensions and restrictions of order continuous functionals, Hokkaida Math. J. 21 (1992), 475-482.

\section{REFERENCES}

[H] W. Hensgen, Contributions to the geometry of vector-valued $H^{\infty}$ and $L^{1} / H_{0}^{1}$ spaces, Habilitation Thesis, Regensburg, 1992.

[HY] E. Hewitt and K. Yosida, Finitely additive measures, Trans. Amer. Math. Soc. 72 (1952), 46-66.

[IT] A. and C. Ionescu-Tulcea, Topics in the theory of lifting, Ergeb. Math. Grenzgeb. (3), vol. 48, Springer, Berlin, 1969.

Universität Regensburg, NAturwissenschaftliche FakUltät I-Mathematik, UniverSITÄTSSTRASSE 31, 93040 REGENSBURG, GERMANY

E-mail address: hensgencalf2.ngate.uni-regensburg.dbp.de 